\title{
Avaliação da capacidade funcional de mulheres com fibromialgia: métodos diretos e autorrelatados
}

\author{
Assessment of functional capacity in women with \\ fibromyalgia: direct and self-reported methods
}

1. Universidade Federal do Paraná. Departamento de Educação Física. Núcleo de pesquisa em qualidade de vida. Curitiba, PR. Brasil

Recebido em 29/09/10 Revisado em 12/01/11 Aprovado em 19/01/11
Resumo - A Fibromialgia (FM) pode limitar a execução das atividades da vida diária (AVD's) e causar impacto negativo na qualidade de vida (QV) destes pacientes. O objetivo deste estudo foi verificar associações entre métodos diretos e indiretos para avaliar a capacidade funcional e a relação destes com algumas características da FM. Participaram do estudo 38 mulheres com diagnóstico de FM. A capacidade funcional foi avaliada através do Health Assessment Questionnaire (HAQ), distância no teste de caminhada de 6 minutos (DTC6) e força de preensão manual (FPM). Aplicou-se o Fibromyalgia Impact Questionnaire (FIQ) para avaliar o impacto na qualidade de vida, a escala visual analógica para mensurar a dor, além da avaliação do índice de massa corporal e circunferência abdominal. Utilizou-se correlação de Pearson para dados paramétricos e de Spearman para não-paramétricos. Além disso, algumas regressões múltiplas foram realizadas. As pacientes apresentaram alta intensidade dolorosa (mediana de 9,5). As correlações encontradas foram: DTC6 versus HAQ ( $\mathrm{r}=-0,55 \mathrm{p}<0,01)$, DTC6 versus FPM $(\mathrm{r}=0,34 \mathrm{p}<0,01)$, dor versus FPM $(\mathrm{r}=-0,41 \mathrm{p}<0,01)$, dor versus HAQ $(\mathrm{r}=0,62 \mathrm{p}<0,01)$ e dor versus FIQ ( $\mathrm{r}=0,66 \mathrm{p}<0,01)$. A intensidade dolorosa explicou uma variação de $40 \%$ na pontuação do HAQ e após a inclusão da DTC6, essa variação aumentou para $60 \%$. Em conclusão, a dor parece comprometer a FPM, as AVD's e a QV destas pacientes. O menor desempenho na DTC6 foi explicado pelo aumento do índice de massa corporal. Em relação à interferência da dor, o HAQ parece ser um instrumento adequado para mensurar a capacidade funcional de mulheres com fibromialgia. Palavras-chave: Dor; Exercíco físico; Obesidade; Qualidade de vida.

Abstract - Fibromyalgia can limit activities of daily living (ADLs) performance and has a negative impact on the quality of life of these patients. The objective of this study was to evaluate the association between direct and indirect methods for the assessment of functional capacity and their relationship with some features of fibromyalgia. Thirty-eight women with a diagnosis of fibromyalgia participated in the study. Functional capacity was assessed by the Health Assessment Questionnaire (HAQ), distance in the six-minute walk test (6MWT), and handgrip strength (HGS). Pain was rated on a visual analog scale. The Fibromyalgia Impact Questionnaire (FIQ) was applied to assess the impact on quality of life. Body mass index and waist circumference were also evaluated. Pearson's correlation test was used for parametric data and Spearman's correlation test for nonparametric data. Multiple regressions were also performed. Pain intensity was high in the patients (median: 9.5). The 6MWT distance was correlated with HAQ score $(r=-0.55, p<0.01)$ and HGS $(r=0.34, p<0.01)$. Pain was correlated with HGS $(r=-0.41$, $p<0.01)$, HAQ score $(r=0.62, p<0.01)$, and FIQ $(r=0.66, p<0.01)$. Pain intensity explained $40 \%$ of the variation in HAQ scores. This variation increased to $60 \%$ after inclusion of $6 \mathrm{MWT}$ distance. In conclusion, pain seems to compromise HGS, ADLs and quality of life in women with fibromyalgia. The lower performance in the 6MWT might be explained by high body mass index. Taking into account pain, the HAQ seems to be an appropriate tool for the assessment of functional capacity in women with fibromyalgia.

Key words: Obesity. Pain; Physical exertion; Quality of Life. 


\section{INTRODUÇÃO}

A Fibromialgia (FM) é uma síndrome caracterizada pela ausência de processo inflamatório e de etiologia pouco esclarecida. O diagnóstico é clinico e baseia-se em dois critérios principais: (1) dor muscular difusa e crônica abaixo e acima da linha da cintura, bilateralmente; (2) hipersensibilidade dolorosa à palpação em pelo menos 11 , dos 18 pontos específicos pré-determinados, também conhecidos como tender points ${ }^{1}$. A FM está associada a diferentes fatores que, isolados ou combinados, favorecem a sua manifestação. Os sintomas de dor crônica, fadiga e sono não-restaurador podem ocasionar alterações de humor e redução do nível de atividade física habitual, o que tende agravar a condição da dor e levar, em casos extremos, à incapacidade funcional ${ }^{2}$.

As repercussões geradas pela dor podem ser inúmeras e a sua presença e a intolerância ao exercício físico, em alguns casos, reprimem a habilidade para o trabalho e para a execução das atividades funcionais cotidianas $^{3}$, acarretando um impacto negativo na qualidade de vida dos pacientes ${ }^{4}$. A funcionalidade nestes indivíduos mostra-se reduzida à medida que estudos demonstram menor força muscular voluntária, capacidade de resistência, habilidade de caminhar, e função dos braços comprometida quando comparados com indivíduos saudáveis ${ }^{5,6}$. Além disso, a maioria das pacientes é sedentária e apresenta excesso de peso? ${ }^{7}$. A FM associada ao índice de massa corporal (IMC) elevado pode relacionar-se ao baixo rendimento físico nessas pacientes ${ }^{8,9}$.

A capacidade funcional pode ser avaliada por instrumentos autorrelatados ou mensurando o desempenho físico. O questionário Health Assessment Questionnaire (HAQ), muito utilizado para avaliação da funcionalidade de pacientes que apresentam doenças reumatológicas ${ }^{10}$, é um instrumento que vem sendo aplicado em pacientes fibromiálgicas ${ }^{8,9}$, porém carece de comparações diretas com testes objetivos.

Testes baseados no desempenho físico são comumente utilizados para discriminar e quantificar dificuldades nas funções corporais ${ }^{6}$. $O$ teste de caminhada de seis minutos (TC6) foi utilizado em estudos anteriores, envolvendo pacientes com FM e apresentou boa confiabilidade ${ }^{6,11}$. O TC6 apresenta boa aplicabilidade, pois ele avalia de forma global a integração de respostas de todos os sistemas fisiológicos envolvidos durante o exercício ${ }^{12}$. Seu ritmo autosselecionado avalia níveis submáximos da capacidade funcional. Muitos indivíduos com alguma limitação de saúde não alcançam capaci- dade máxima de exercício durante sua realização e devido a maioria das atividades da vida diária (AVD's) serem realizadas em níveis submáximos de esforço, a distância caminhada durante o TC6 pode melhor refletir a capacidade funcional para as AVD's' ${ }^{12,13}$.

A força de preensão manual (FPM), em populações mais frágeis, como de idosos, correlaciona-se com uma série de testes funcionais e sua medida não deve ser descartada devido as suas vantagens como portabilidade, baixo custo, boa reprodutibilidade, dentre outras ${ }^{14}$. Níveis baixos de FPM estão associados com uma maior propensão de mortalidade prematura e o desenvolvimento de incapacidades ${ }^{15}$.

O TC6 e a dinamometria discriminam pacientes com fibromialgia e indivíduos saudáveis ${ }^{5}$, além de se correlacionarem melhor com vários instrumentos autorrelatados para avaliar a capacidade funcional ${ }^{6}$. Entretanto, a relação entre a funcionalidade mensurada por testes diretos e por escalas classificatórias tem sido pouco investigada em pacientes fibromiálgicos ${ }^{6}$.

A obtenção de parâmetros físicos e autorrelatos da capacidade funcional tornam-se importantes para verificar possíveis limitações funcionais já existentes, além de diagnosticar a funcionalidade por diferentes meios. Assim, o objetivo deste estudo foi avaliar a capacidade funcional de mulheres com FM, utilizando o TC6, a dinamometria e o questionário HAQ, verificando associações entre funcionalidade, variáveis antropométricas, dor e qualidade de vida.

\section{PROCEDIMENTOS METODOLÓGICOS}

Tipo de estudo e identificação dos sujeitos Estudo transversal, descritivo e correlacional, aprovado pelo Comitê de Ética em seres humanos do Hospital de Clínicas da Universidade Federal do Paraná (HC/UFPR), Curitiba, Paraná, seguindo as diretrizes propostas na resolução 196/96 do conselho nacional de saúde sobre pesquisas, envolvendo seres humanos, registrado sob o número $\mathrm{CEP} / \mathrm{HC}$ : 1469.137/2007-06.

Após consulta com médicos especialistas do Ambulatório de Reumatologia do HC/UFPR, pacientes apresentando diagnóstico de fibromialgia, conforme o Colégio Americano de Reumatologia ${ }^{1}$ foram convidadas a participar do estudo.

\section{Critérios de inclusão e exclusão}

Foram incluídas mulheres apresentando diagnóstico de fibromialgia, com idade entre 20 e 64 anos, 
IMC entre 18,5 e $39,9 \mathrm{~kg} / \mathrm{m}^{2}$ e que conseguissem, minimamente, realizar as AVD's. Pacientes com limitações funcionais graves e que utilizavam algum dispositivo de auxilio para o desempenho de suas atividades diárias foram excluídas do estudo. Todas estas informações foram obtidas através do autorrelato e do prontuário das pacientes. Um total de 43 mulheres aceitou o convite e após a aplicação dos critérios de inclusão e exclusão, a amostra final foi composta por 38 indivíduos.

Depois da obtenção da assinatura do termo de consentimento livre e esclarecido, foram realizadas avaliação antropométrica, avaliação da capacidade funcional e avaliação do impacto da FM na qualidade de vida dessas pacientes.

\section{Avaliação antropométrica}

Foram mensuradas a massa corporal $(\mathrm{kg})$ por meio de balança digital (Plenna®, modelo Sport Branca), e a estatura $(\mathrm{cm})$ por meio de estadiômetro fixo na parede (Wiso ${ }^{\circledR}$, modelo compacto 02Mts), conforme o Anthropometric Standardization Reference Manual $^{16}$, para a obtenção do IMC, que foi classificado segundo a Organização Mundial da Saúde $(\mathrm{OMS})^{17}$. A circunferência abdominal foi avaliada com fita antropométrica flexível e inextensível (Gulick-Mabbis ${ }^{\circledR}$ ). Classificou-se a obesidade abdominal para os valores iguais ou acima de $80 \mathrm{~cm}$ conforme proposto pela $\mathrm{OMS}^{17}$.

\section{Avaliação da capacidade funcional e impacto da FM na qualidade de vida}

A capacidade funcional foi avaliada por testes físicos que envolveram os membros superiores (dinamometria) e membros inferiores (TC6), além da aplicação do HAQ. O impacto da FM na qualidade de vida foi mensurado através da aplicação do questionário FIQ.

O TC6 foi realizado em um corredor plano, apresentando as dimensões de 30 metros de comprimento por 1,5 metros de largura. Os pacientes foram instruídos a caminhar a maior distância possível, numa velocidade confortável, indo e vindo por este corredor até esgotar o tempo de 6 minutos, podendo interromper o teste a qualquer momento, quantas vezes fosse necessário, caso houvesse algum desconforto, com o cronômetro permanecendo ligado e depois, retornassem para terminar o teste ao fim dos 6 minutos estipulados. Registrou-se a distância percorrida (DTC6) em metros ao final de cada teste ${ }^{12}$. Inicialmente, com 15 avaliadas, foram realizados dois testes, com intervalo de 1 semana, e os resultados não apresentaram diferenças estatis- ticamente significativas (teste 1: 477,06 $\pm 111,17 \mathrm{~m}$ versus teste 2: 493,59 $\pm 77,68 \mathrm{~m}, \mathrm{p}=0,43)$. Desta forma, achamos conveniente realizar apenas 1 teste.

Foi utilizado um dinamômetro hidráulico manual (JAMAR Hydraulic Hand Dynamometer, Modelo PC-5030J1, Sammons Preston Rolyan, Inc. USA) para avaliar a FPM. Este instrumento mostra-se válido por possuir uma boa confiabilidade ${ }^{18}$. Ele foi posicionado ao lado do corpo, estando o individuo sentado, com o braço totalmente estendido, apontando o aparelho para a superfície terrestre onde o avaliado foi instruído a apertá-lo durante cerca de 2-3 segundos. Este protocolo é uma adaptação proposta pela American Society of Hand Therapists ${ }^{19}$. No presente estudo, o indivíduo manteve o braço totalmente estendido diferentemente da proposta original que recomenda o cotovelo estar flexionado à $90^{\circ}$, porém existem resultados conflitantes quanto à posição do cotovelo na geração de força ${ }^{20}$. Muitas pacientes relataram que o protocolo com o braço estendido era mais confortável para a realização do teste. Em ambos os membros superiores, repetiu-se este procedimento três vezes, alternadamente. Considerou-se o maior valor obtido ao final das três séries executadas, adotando o valor referente ao membro superior predominante. Antes do início do teste, cada individuo foi solicitado a realizar 2 repetições em cada um dos lados como forma de adaptação.

A capacidade funcional mensurada de forma subjetiva foi avaliada através da aplicação do Health Assessment Questionnaire (HAQ) em sua versão traduzida e validada para o Brasil ${ }^{21}$. Ele é dividido em oito componentes: vestir-se, levantar-se, comer, caminhar, higienizar-se, alcançar, preensão manual e outras atividades. Cada componente contém duas ou três questões, perfazendo um total de 20 questões. Cada questão apresenta quatro opções como resposta (zero a três) e o individuo deve assinalar uma delas. Sua pontuação varia de zero a três e quanto maior a pontuação no HAQ, maior significa a incapacidade no sujeito. Pontuação final de 0 a 1 geralmente representa dificuldade leve a moderada, 1 a 2 representa dificuldade moderada a incapacidade grave e 2 a 3 indica incapacidade grave a muito grave ${ }^{10}$.

Utilizou-se o Fibromyalgia Impact Questionnaire (FIQ), traduzido e validado para o Brasil ${ }^{22}$, para avaliação do impacto desta síndrome na qualidade de vida destas pacientes. Sua pontuação varia de zero a 100 e quanto mais alta, maior o impacto na qualidade de vida. Neste estudo, optou-se por utilizar o primeiro componente do FIQ também 
para avaliar a capacidade funcional das pacientes. Suas perguntas informam com que frequência as pacientes desempenharam as atividades da vida diária. O emprego de sua pontuação de forma isolada à pontuação total do questionário FIQ não é validada, porém, a sua aplicação vem ocorrendo em diversos estudos ${ }^{6,11}$. Denominou-se sua utilização neste estudo como FIQ-CF. A questão cinco do FIQ (escala visual analógica, 0-10) foi aplicada para avaliar a dor. Tanto a aplicação do HAQ quanto do FIQ foi realizada em forma de entrevista.

\section{Tratamento Estatístico}

Os dados foram analisados pelo programa Minitab Statistical Software (versão 15.0), utilizando para a normalidade dos dados o teste de Shapiro-Wilk. Utilizaram-se a correlação de Pearson para os dados paramétricos e de Spearman para os dados não-paramétricos com um intervalo de confiança de 95\%, além da análise de regressão múltipla para verificar a associação entre os testes funcionais (DTC6, FPM e HAQ) com as variáveis (idade, IMC, dor e FIQ).

\section{RESULTADOS}

As características gerais da amostra são apresentadas na Tabela 1. Verificaram-se 36,80\% (n=14) de sobrepeso e 44,70\% (n=17) de obesidade. Levando em consideração o excesso de gordura abdominal,
94,70\% ( $n=36)$ apresentaram valores acima do normal em relação à circunferência abdominal.

Tabela 1. Características demográficas e antropométricas da amostra

\begin{tabular}{lccc}
\hline Variáveis & Média + DP & Mínimo & Máximo \\
\hline Idade & $47,15 \pm 8,09$ & 29 & 62 \\
Peso $(\mathrm{kg})$ & $73,40 \pm 11,53$ & 49,60 & 97,20 \\
Estatura $(\mathrm{cm})$ & $157,28 \pm 6,54$ & 145,50 & 169,70 \\
IMC $\left(\mathrm{kg} / \mathrm{m}^{2}\right)$ & $29,73 \pm 4,87$ & 20,67 & 39,90 \\
CA $(\mathrm{cm})$ & $95,30 \pm 10,81$ & 75,00 & 121,00 \\
\hline
\end{tabular}

Os dados referentes às variáveis estudadas são apresentados na Tabela 2. A pontuação média do HAQ foi $1,75 \pm 0,51$ e mediana 1,81 , valores estes que representam uma dificuldade moderada à incapacidade grave. As correlações realizadas entre todas as variáveis são apresentadas na Tabela 3

A Tabela 4 apresenta os resultados da análise de regressão múltipla realizada na qual foram escolhidas como variáveis independentes a idade, IMC, impacto da fibromialgia na qualidade de vida (FIQ) e a dor, e como variáveis dependentes a DTC6, a FPM e a pontuação do questionário HAQ. As variáveis independentes explicaram uma variação de $17-40 \%$ em relação aos resultados para a capacidade funcional. Acrescentando a FPM e a DTC6, como variáveis independentes e mantendo o HAQ como variável dependente, verificou-se que

Tabela 2. Dados referentes à capacidade funcional, intensidade dolorosa e impacto da FM na qualidade de vida da amostra estudada.

\begin{tabular}{|c|c|c|c|c|}
\hline Variáveis & Média + DP & Mediana & Mínimo & Máximo \\
\hline DTC6 (m) & $472,87 \pm 69,95$ & 480 & 320,00 & 625,00 \\
\hline FPM (kgf) & $23,36 \pm 8,97$ & 22,50 & 6,00 & 40,00 \\
\hline FIQ-CF (0-10) & $5,27 \pm 2,07$ & 5,32 & 1,66 & 9,66 \\
\hline $\operatorname{DOR}(0-10)$ & $8,65 \pm 1,64$ & 9,5 & 5 & 10 \\
\hline
\end{tabular}

Tabela 3. Correlações das variáveis estudadas.

\begin{tabular}{|c|c|c|c|c|c|c|c|c|c|}
\hline & Idade & IMC & $\mathrm{CA}$ & DTC6 & FPM & HAQ & FIQ-CF & FIQ & Dor \\
\hline Idade & 1 & & & & & & & & \\
\hline IMC & 0,23 & 1 & & & & & & & \\
\hline DTC6 & $-0,21$ & $-0,38$ & $-0,40$ & 1 & & & & & \\
\hline FPM & $-0,12$ & $-0,30$ & $-0,34$ & $0,34^{* *}$ & 1 & & & & \\
\hline FIQ-CF & 0,07 & 0,17 & 0,25 & $-0,44^{* *}$ & $-0,41 *$ & $0,48 * *$ & 1 & & \\
\hline FIQ & 0,15 & 0,05 & 0,07 & $-0,11$ & $-0,43 * *$ & $0,40 *$ & $0,49 * *$ & 1 & \\
\hline Dor & 0,20 & 0,12 & 0,12 & $-0,16$ & $-0,41 * *$ & $0,62 * *$ & $0,41 *$ & $0,66 * *$ & 1 \\
\hline
\end{tabular}

*Correlação significante ao nível de $\mathrm{p} \leq 0,05$

** Correlação significante ao nível de $p \leq 0,01$ 
Tabela 4. Análise de regressão múltipla, considerando as variáveis investigadas.

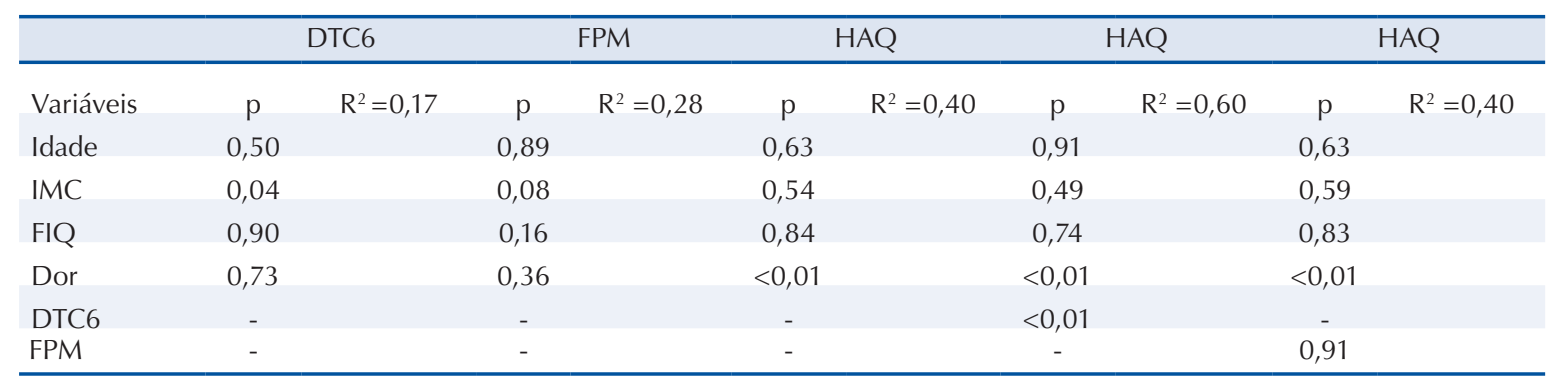

somente a DTC6 influenciou na pontuação deste questionário. Neste caso, a dor e a DTC6 foram as únicas variáveis que apresentaram resultados significativos na variação do HAQ.

Apesar de uma alta prevalência de excesso de peso e obesidade visceral, nenhuma dessas duas variáveis correlacionou-se de forma direta com as outras variáveis analisadas. Porém, o IMC foi a variável significativa que contribuiu para maior variação em relação a menores distâncias caminhadas no TC6.

\section{DISCUSSÃO}

A avaliação funcional busca informações clínicas e objetivas que servem para direcionar um tratamento adequado, que pode impedir ou retardar o processo de incapacidade das pacientes com FM.

A dor é uma característica debilitante e pode ser considerada como uma das principais causas de incapacidade, podendo comprometer a qualidade de vida destes pacientes. Neste estudo, verificou-se correlação moderadamente alta e significativa $(\mathrm{r}=0,66)$ entre a dor e o impacto da fibromialgia na qualidade de vida. Além disso, houve outra correlação moderadamente alta e significativa $(r=0,62)$ entre a variável dor e o questionário $\mathrm{HAQ}$, utilizado para verificar o nível de capacidade funcional dos indivíduos através de atividades da vida diária desempenhadas, resultados similares aos encontrados por Guler e Sahin?.

Verificou-se, neste estudo, que a dor também apresentou uma significativa correlação com a FPM $(\mathrm{r}=-0,41)$. Sahin et al. ${ }^{23}$ encontraram diferenças estatisticamente significativas entre o grupo de mulheres saudáveis e o grupo FM em relação à força muscular. O resultado encontrado no presente estudo $(23,36 \pm 8,97 \mathrm{kgf})$ para essas pacientes é levemente maior, comparado ao estudo anterior, porém inferior a média encontrada para uma amostra de 400 mulheres brasileiras saudáveis (31,6 kgf para o lado dominante e $28,4 \mathrm{kgf}$ para o lado não-dominante) ${ }^{24}$. Valores próximos de 20 kgf estão relacionados de forma independente para riscos de dependência futura e baixos níveis de saúde ${ }^{25}$.

Entre os questionários utilizados para avaliar a capacidade funcional (HAQ e FIQ-CF) houve uma significativa e baixa correlação $(r=0,48)$. Entre os testes realizados para avaliar o desempenho físico (TC6 e dinamometria) encontrou-se, novamente, uma baixa $(\mathrm{r}=0,34)$ e significativa correlação, indicando que as pacientes que apresentaram menor força dos membros superiores caminharam menores distâncias, corroborando Mannerkorpi et al. ${ }^{6}$.

O questionário HAQ apresentou correlação significativa apenas com a DTC6 $(\mathrm{r}=-0,55)$, porém o mesmo não foi observado com a FPM. A parte específica do questionário FIQ, para medir a capacidade funcional (FIQ-CF), apresentou correlações significativas tanto com a DTC6 quanto com a FPM. Mesmo a primeira questão do FIQ não sendo validada como um instrumento para medir a capacidade funcional, um estudo anterior também mostrou correlações entre FIQ-CF e DTC6 e FIQ-CF e FPM ${ }^{6}$. Apesar destes resultados encontrados, deve ser ressaltado que o FIQ-CF não é um instrumento validado, apenas um componente de um questionário mais abrangente.

A avaliação da capacidade funcional, utilizando o Health Assessment Questionnaire em pacientes com FM, na literatura, não é novidade ${ }^{8,9}$, porém a sua associação com testes diretos como o TC6 e com a dinamometria ao nosso conhecimento não existe. A associação do HAQ encontrada com a DTC6, além da maior variação de sua pontuação ser explicada pela intensidade dolorosa sentida pelas pacientes é um indicativo de que seu uso pode ser bem aplicado para medir a capacidade funcional de pacientes com fibromialgia.

Esperava-se que a dor pudesse influenciar de forma significativa o desempenho no TC6, porém não foi isso o que se verificou. Mesmo assim, a baixa distância percorrida pode ser um indicativo de um comprometimento da capacidade funcional e isso pôde ser demonstrado neste estudo pela correlação moderada e negativa encontrada entre o HAQ e a 
DTC6. Além disso, a dor e a DTC6 foram as únicas variáveis que influenciaram de forma significativa a variação da pontuação do HAQ, explicando uma variação de $60 \%$. Isso demonstra o quanto a limitação para uma atividade básica, como a caminhada, pode interferir no desempenho das demais atividades da vida diária.

A distância percorrida pelas pacientes pode ser comparada com outros estudos. Pankoff et al. ${ }^{11}$ , realizando três vezes o TC6 com cada paciente (27-59 anos de idade), encontraram valores de 478 $\pm 61 \mathrm{~m}, 492 \pm 57 \mathrm{~m}$ e $495 \pm 60 \mathrm{~m}$ respectivamente. Ainda, Panton et al. ${ }^{26}$, comparando mulheres saudáveis, mulheres idosas saudáveis e mulheres com FM, verificaram valores de distâncias percorridas de $603,5 \pm 134,2 \mathrm{~m}, 507,8 \pm 102,1 \mathrm{~m}$ e 486,8 $\pm 82,5 \mathrm{~m}$, respectivamente, entre estes três grupos. Observa-se que pacientes com FM, geralmente percorrem distâncias menores do que outros grupos estudados.

Uma característica bem marcante também nestes pacientes é que a maioria dos indivíduos apresenta sobrepeso ou obesidade. No atual estudo, também encontrou-se um alto percentual $(81,50 \%)$ para excesso de peso, prevalência esta maior que os $73 \%$ encontrados recentemente ${ }^{27}$. Yunus et al. ${ }^{8}$ afirmam que esta síndrome está relacionada diretamente com um baixo rendimento físico nesses pacientes, enquanto Guler e Sahin ${ }^{9}$ relatam que este baixo rendimento físico está simplesmente associado com o IMC elevado nestes mesmos indivíduos. Em seu estudo, Yunus et al..$^{8}$ encontraram uma associação significativa entre o HAQ e IMC, porém, no presente estudo, isso não foi confirmado. Em relação aos dois testes físicos, mesmo que não tenha sido encontrada uma relação direta significativa do IMC com a DTC6 e com a FPM, o índice de massa corporal das pacientes avaliadas neste estudo parece contribuir de forma significativa para a variação de desempenho de, pelo menos, um dos testes (DTC6), já a dor não.

No presente estudo, podemos verificar que a dor contribuiu de forma significativa para o desempenho nas atividades da vida diária e por isso o Health Assessment Questionnaire pode ser utilizado como uma forma de verificar a funcionalidade destas pacientes em função da dor, já que esta é a principal característica da fibromialgia.

Uma limitação deste estudo foi a de não ter avaliado o nível de atividade física (NAF). Sabe-se que o exercício físico é crucial como parte do tratamento destes pacientes e evidências recentes mostram benefícios moderados dos exercícios físicos, sobretudo os aeróbicos, na capacidade funcional e possivelmente na dor ${ }^{28}$. Apesar destes achados na literatura, é difícil afirmar que o NAF, no presente estudo, pôde ter influenciado nas associações entre dor e capacidade funcional. Pouco tem sido pesquisado sobre a quantificação e caracterização do NAF nestes pacientes e a maioria dos estudos utiliza instrumentos autorrelatados. Porém, a necessidade por uma medida objetiva é essencial ${ }^{29}$.

Os acelerômetros oferecem muitas vantagens sobre as técnicas autorrelatadas ${ }^{29}$ e podem ser considerados a medida mais adequada para tal propósito. A recomendação existente é utilizá-lo ao nível da cintura ${ }^{29}$, porém em pacientes adultos com fibromialgia apenas um estudo, e recente ${ }^{30}$, se propôs a tal finalidade. Isto é um indicativo de quanto limitado é caracterizar a associação do NAF com a dor. Os próprios autores do estudo, citado acima ${ }^{30}$, assumem que não há relação entre essas duas variáveis. Futuras pesquisas podem investigar melhor a relação entre nível de atividade física, dor e capacidade funcional.

\section{CONCLUSÕES}

A dor parece comprometer a força de preensão manual, o desempenho para as atividades cotidianas e a qualidade de vida destas pacientes. $\mathrm{O}$ excesso de peso foi responsável por contribuir para a variação no desempenho no teste de caminhada de 6 minutos, porém a dor não.

O questionário Health Assessment Questionnaire mostrou-se mais eficiente na avaliação da capacidade funcional feita de forma subjetiva do que o componente específico para esta finalidade do questionário Fibromyalgia Impact Questionnaire. Além disso, o estudo mostrou que as pacientes que apresentaram maior dificuldade na realização das tarefas da vida diária acabaram caminhando menores distâncias. Em relação à interferência da dor, o Health Assessment Questionnaire parece ser mais viável para mensurar a capacidade funcional de mulheres com fibromialgia.

\section{Agradecimentos}

Ao Conselho Nacional de Desenvolvimento Cientifico e Tecnológico (CNPq) pela concessão da bolsa de iniciação cientifica ao aluno/pesquisador Diogo Homann.

\section{REFERÊNCIAS BIBLIOGRÁFICAS}

1. Wolfe F, Smythe HA, Yunus MB, Bennet RM, Bombardier C, Goldenberg DL, et al. The American College of Rheumatology 1990 criteria for the classification of fibromyalgia: report of the multicenter criteria committee. Arthritis Rheum 1990;33(2):160-72. 
2. Bernard AL, Prince A, Edsall P. Quality of life issues for fibromyalgia patients. Arthritis Care Res 2000;13(1):42-50.

3. Jacobsen S, Petersen IS, Daneskiold-Samsoe B. Clinical features in patients with chronic muscle pain: with special reference to fibromyalgia. Scand J Rheumatol 1993;22(2):69-76.

4. Martinez JE, Ferraz MB, Sato EI, Atra E. Fibromyalgia versus rheumatoid arthritis: a longitudinal comparison of the quality of life. J Rheumatol 1995;22(2):270-4

5. Mannerkorpi K, Svantesson U, Carlsson J, Ekdahl C. Tests of functional limitations in fibromyalgia syndrome: a reliability study. Arthritis Care Res 1999;12(3):193-9.

6. Mannerkorpi K, Svantesson U, Broberg C. Relationships between performance-based tests and patients' ratings of activity limitations, self-efficacy, and pain in fibromyalgia. Arch Phys Med Rehabil 2006;87(2):259-64.

7. Bennett RM, Jones J, Turk DC, Russell IJ, Matallana L. An internet survey of 2,596 people with fibromyalgia. BMC Musculoskelet Disord 2007;8:27. doi:10.1186/14712474-8-27.

8. Yunus MB, Arslan S, Aldag JC. Relationship between body mass index and fibromyalgia features. Scand J Rheumatol 2002;31(1):27-31

9. Guler H, Sahin G, As I. Fibromyalgia, obesity and obesity related measurements. The Pain Clinic 2006;18(1):25-9.

10. Bruce B; Fries JF. The Health Assessment Questionnaire (HAQ). Clin Exp Rheumatol 2005;23(5 Suppl. 39):S14-8.

11. Pankoff BA, Overend TJ, Lucy SD, White KP. Reliability of the six-minute walk test in people with fibromyalgia. Arthritis Care Res 2000;13(5):291-5.

12. American Thoracic Society. ATS Statement: Guidelines for the Six-Minute Walk Test. Am J Respir Crit Care Med 2002;166(1):111-7.

13. Du H, Newton PJ, Salamonson Y, Carrieri-Kohlman VL, Davidson PM. A review of the six-minute walk test: its implication as a self-administered assessment tool. Eur J Cardiovasc Nurs 2009;8(1):2-8.

14. Geraldes AAR, Oliveira ARM, Albuquerque RB, Carvalho JM, Farinatti PTV. A força de preensão manual é boa preditora do desempenho funcional de idosos frágeis: um estudo correlacional múltiplo. Rev Bras Med Esporte 2008;14(1):12-6.

15. Bohannon RW Hand-grip dynamometry predicts future outcomes in aging adults. J Geriatr Phys Ther 2008;31(1):3-10.

16. Lohman TG, Roche AF, Martorel R. Anthropometrics standartization reference manual. Champaign: Human Kinetics Books; 1988.

17. World Health Organization. Obesity: preventing and manging the global epidemic. Report of a WHO consultation on obesity. Geneva:2000

18. Figueiredo IM, Sampaio RF, Mancini MC, Silva FCM, Souza MAP. Teste de força de preensão utilizando o dinamômetro Jamar. Acta Fisiátrica 2007;14(2):104-10
19. Richards LG, Olson B, Pamiter-Thomas P. How forearms position affects grip strength. Am J Occup Ther 1996;50(2):133-8.

20. Innes E. Handgrip strength testing: $A$ review of the literature. Aust Occup Ther J 1999;46(3):120-40

21. Ferraz MB, Oliveira LM, Araujo PM, Atra E, Tugwell P. Cross-cultural reliability of the physical ability dimension of the health assessment questionnaire. J Rheumatol 1990;17(6):813-7

22. Marques AP, Santos AMB, Assumpcao A, Matsutani LA, Lage LV, Pereira CAB. Validação da versão brasileira do Fibromyalgia Impact Questionnaire (FIQ). Rev Bras Reumatol 2006;46(1):24-31.

23. Sahin G, Ulubas B, Calikoglu M, Erdogan C. Handgrip strength, pulmonary function tests, and pulmonary muscle strength in fibromyalgia syndrome: Is there any relationship? South Med J 2004;97(1):25-9.

24. Caporrino FA, Faloppa F, Santos JBG, Réssio C, Soares FHC, Nakachima LR et al. Estudo populacional da força de preensão palmar com dinamômetro Jamar. Rev Bras Ortop 1998;33(2):150-4

25. Jylha M, Guralnik JM, Balfour J, Fried LP. Walking difficulty, walking speed, and age as predictors of self-rated health: the women's health and aging study. J Gerontol A Biol Sci Med Sci 2001;56(10):M609-17.

26. Panton LB, Kingsley JD, Toole T, Cress ME, Abboud G, Sirithienthad P, et al. A Comparison of Physical Functional Performance and Strength in Women With Fibromyalgia, age and weigh matched controls, and older women who are healthy. Phys Ther 2006;86(11):1479-88

27. Neuman L, Lerner E, Glazer Y, Bolotin A, Shefer A, Buskila D. A cross-sectional study of the relationship between body mass index and clinical characteristics, tenderness measures, quality of life, and physical functioning in fibromyalgia patients. Clin Rheumatol 2008;27(12):1543-7

28. Thomas EN, Blotman F. Aerobic exercise in fibromyalgia: a practical review. Rheumatol Int 2010;30(9):1143-50

29. Ward DS, Evenson KR, Vaughn A, Rodgers AB, Troiano RP. Accelerometer use in physical activity: best practices and research recommendations Med Sci Sports Exerc 2005;37(11 Suppl.):S582-8.

30. McLoughlin MJ, Colbert LH, Stegner AJ, Cook DB. Are women with fibromyalgia less physically active than healthy women? Med Sci Sports Exerc 2011;43(5):905-12.

\author{
Endereço para correspondência \\ Diogo Homann \\ Universidade Federal do Paraná. Departamento \\ de Educação Física,. \\ Rua Coração de Maria, 92 (BR-116, km 92). \\ Jardim Botânico \\ CEP 80215-370 - Curitiba, PR. Brasil \\ E-mail: diogomann@hotmail.com
}

